\title{
Exploring Whole-Hand Gestures in a Tabletop Environment for Urban Modeling
}

\author{
Peter Vandoren, Karel Frederix, Karin Coninx, and Frank Van Reeth \\ Hasselt University - tUL - IBBT, Expertise Centre for Digital Media, \\ Wetenschapspark 2, 3590 Diepenbeek, Belgium \\ \{firstname. lastname\} @uhasselt.be
}

\begin{abstract}
Touch-based tabletop interaction with virtual environments (VEs) is a recent research interest. In particular, 3D navigation and object interaction on tabletops pose considerable research challenges given the flat, rectangular twodimensional workspace of an interactive tabletop. This paper explores the design of whole-hand gestures for urban modeling on an interactive, tilted tabletop system. These touch-based gestures fit a hybrid 2D/3D approach for navigation and object interaction in urban city modeling. In a formal user study the proposed whole-hand gestural interface was compared to a finger-based equivalent. The evaluation results reveal appreciation for aspects of the interaction concept, also illustrating the need for further tuning of the interaction concept and the input tracking.
\end{abstract}

Keywords: interaction techniques, tabletop, gestures, touch-based interaction, navigation, urban modeling, user evaluation.

\section{Introduction}

The emergence of multi-touch surfaces $[1,2,3]$ has been quite a boost for research in touch-based interaction in the past decade. In 2007 Grossman [4] presented an overview of research advances in interaction techniques on interactive tabletops, indicating that three-dimensional interaction was largely undeveloped. Several novel techniques for 3D interaction have since been presented, either simulating 3D interaction with finger-based interaction while touching the surface $[5,6,7,8]$ or above surface interaction using vision or additional sensors [9, 10, 11].

Our approach explores interaction on the surface using whole-hand gestures, motivated by the versatility and the expressiveness of the human hand. We developed a set of novel gestures for navigating in a virtual environment and for modeling 3D objects (in our case, buildings in a virtual city). In the next sections we describe the interaction design and elaborate on the results of an initial user study, comparing whole-hand interaction and finger-based interaction.

\section{Whole-Hand Gesturing in Urban Modeling}

The extensive experience of regular computer users with point-oriented, direct interaction techniques in 2D desktop applications generally results in a smooth 
transition towards finger-based interaction on tabletops. Although people are proficient in whole-hand interaction in the real world, this kind of interaction is much less exploited in tabletop environments. Reasons for this absence include tracking issues and a rather large occlusion area, but the lack of suitable interaction metaphors might be one as well.

We explored these issues in the TT-GUM (TableTop Gestures for Urban Modeling) platform, developed on an interactive touch-sensitive surface using frustrated total internal reflection (FTIR) [3]. The surface, measuring 80 by 60 centimeter, is tilted $45^{\circ}$ to facilitate hand-based gestures. TT-GUM (fig. 1) offers two perspective views on the virtual city, a frontal view and a top view. The user switches to the desired view by touching a virtual button, located on the top right-hand side of the surface. TT-GUM offers gestures for navigation and object modeling in two separate interaction modes in order to reduce the complexity of the gestural interaction.

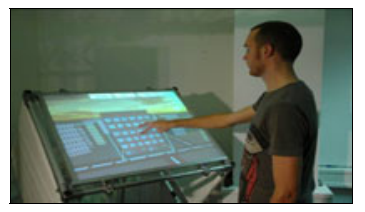

Fig. 1. The TT-GUM platform

The design of gestures for navigation in the virtual city is inspired by the movements of the Harrier jet aircraft [12], which is also able to perform typical helicopter actions (vertical take-off and hover). The basic posture for navigation is a flat hand that is positioned upright on the surface (fig. 2) and symbolizes the fuselage of the Harrier.

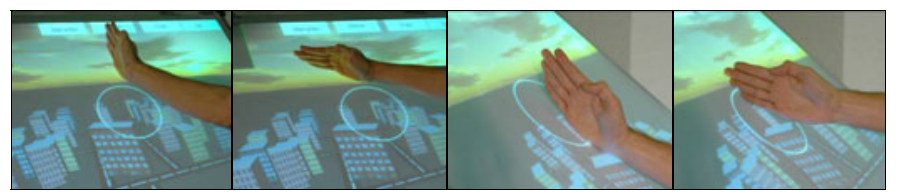

Fig. 2. Navigation a) forward b) turning left c) climbing d) descending

Pushing the hand forward initiates a forward travel of the virtual camera (fig. 2.a). If the hand is positioned in an angle with regard to the straight ahead position, the virtual camera turns in the direction indicated by the hand (fig. 2.b). Changing altitude of the virtual camera is achieved by altering the contact surface of the hand with the table. Lifting the front side of the hand, while keeping the back end on the table, results in a climb (fig. 2.c). Lifting the backside, while the front end of the hand remains on the table, commands the camera to descend (fig. 2.d). The white circle in the center of the surface defines a neutral area for horizontal camera travel, allowing only panning or changing the altitude.

The concept for object modeling in TT-GUM is also based on whole-hand interaction. Object are moved (fig. 3.a) by positioning (two or) three fingers (normally 
the three central fingers of the hand) on a building and dragging the building to the intended place. Positioning at least four fingers of the hand on or near a building initiates rotation (fig. 3.b). As it turns out that the little finger often lifts from the surface when a rather large rotating move (several tens of degrees) is made, the rotation requires at least a four-finger contact. Especially when using the top view on the virtual city, the similarity between the proposed rotation gesture and rotating a physical object emerges. Scaling a virtual building can be performed in one or two dimensions. The basic idea is that by positioning a flat hand on the edge of a building (fig. 3.c), the hand will stick to that edge as long as it touches the surface; so by moving the hand parallel to the edge, the building is scaled according to the movement. This technique, defined analogous to the "sticky finger" technique by Pierce [13], was extended in a dual dimension scaling gesture. By placing a cornering on the corner of a building (fig. 3.d), that corner sticks to the hand shape and hence enables concurrent scaling in two dimensions.
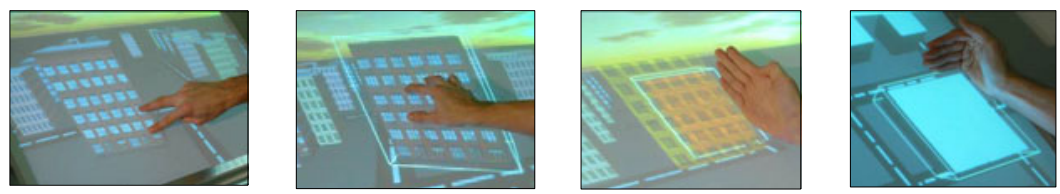

Fig. 3. Object manipulation: a) moving b) rotating c) scaling d) two-dimensional scaling

\section{User Experiment}

\subsection{Design and Setup}

We organized a comparative user study, to gain insight in the value of the proposed whole-hand gestures and to receive subjective feedback from the test participants. We defined a finger-based gestural interaction equivalent, being touch-based and using at most 2 fingers to perform navigation or object manipulation, as a basis of comparison. The user population consisted of sixteen persons, all computer scientists, split into 2 groups based on their interaction experience with VEs. We defined a within-subjects test design, counter balanced on the interaction technique. Independent variables were the interaction technique, the presence of the wireframe aura representation around a building and the density of buildings in the virtual city. A test session for an interaction technique comprised a sequence of 15 navigational tasks, followed by three sequences of 20 object docking tasks each for move, rotate and scale operations. We scheduled the test conditions in a Latin square design to neutralize the effects of learning. After the test, the participants were asked to fill out a questionnaire.

\subsection{Test Results and Discussion}

The finger-based interaction performed statistically significant faster than whole-hand interaction (using ANOVA, p<0.05), for navigation as well as object manipulation. With regard to the effect of city density or the use of a wireframe aura representation, 
we can't report any statistically significant difference in task execution times. The subjective results indicate that the participants are convinced that finger-based interaction is faster and more precise. Finger-based interaction is generally considered to be more intuitive than hand-based interaction. When looking at the user preference for individual operations, 3 out of 16 users preferred travelling using whole-hand navigation, whereas 7 users preferred the panning operation in whole-hand navigation. We found similar results with regard to object manipulation: 3 participants preferred the translation using whole-hand interaction, whereas half of the participants opted for rotation according to whole-hand interaction and a quarter of the test group chose whole-hand interaction for scaling.

Eleven participants mentioned some kind of frustration with whole-hand interaction, as the system did not react to their expectations when gesturing. Although the majority of the reported issues were due to incorrect gesturing by the participants, the problems of 4 participants were a consequence of above average differences in the hand shapes of these users and should be solved by updating the hand shape calibration in the touch tracking software. The height of the test person and the arms' reach is also a matter to take into further consideration: 3 participants, being smaller than 1.7 meter, had some difficulty when scaling the height of a building as their hand was not correctly positioned along the top edge of the building because of insufficient arms' reach.

Several factors, probably in a combination, could be responsible for the considerable difference in task completion time between finger-based navigation and whole-hand navigation. A first reason of this difference is that hand-based navigation is a more continuous action, hence requiring also more consciously calculating, in contrast to the sequence of discrete actions in finger-based navigation. A second factor is the presence of a sideward move in finger-based navigation, which was not available in whole-hand navigation because of the airplane metaphor. An additional element is that hand-based navigation was more difficult to master than finger-based navigation, as substantiated by the larger learning curve of whole-hand navigation over the 15 navigational tasks.

About half of the test population caused accidental moves of buildings when using whole-hand manipulation as they "released" the building incorrectly, by lifting the fingers not synchronously from the surface or by slightly dragging across the surface when lifting their hand. This situation will have led to greater attention and caution while performing the tasks using whole-hand interaction, at least with the users that weren't able to master the gesture completely. Hence one might conclude that wholehand interaction was also more difficult to learn, albeit that 7 test participants managed to rotate buildings on average faster with whole-hand interaction than with finger-based interaction. As the whole-hand gesture for rotation has many similarities with rotating physical 3D objects on a table and as it supports proprioception, many users might have felt quite comfortable with this gesture and might therefore have expressed their preference for this gesture.

\section{Conclusions and Future Work}

This paper presented whole-hand gestures for hybrid 2D/3D navigation and object manipulation in a tabletop environment. A comparative user study showed that, given 
the current gesture design and implementation, finger-based interaction maintains the efficiency advantage. Most participants preferred finger-based interaction in general, but up to half of the participants expressed a preference for certain hand-based gestures and in particular for rotation. Besides enhancing the interaction design and the input tracking, we will perform further research on the application context where either finger-based interaction or whole-hand gestures are beneficial.

Acknowledgements. The authors would like to thank their former colleagues, Rohita Khatiwada and Chris Raymaekers, for their valuable contributions to this research. Part of this research was performed in the context of the IBBT-project HiMasquerade.

\section{References}

1. Dietz, P., Leigh, D.: DiamondTouch: a Multi-user Touch Technology. In: 14th ACM Symposium on User Interface Software and Technology, pp. 219-226. ACM Press, New York (2001)

2. Microsoft Surface, http://www.microsoft.com/surface/

3. Han, J.Y.: Low-cost Multi-touch Sensing through Frustrated Total Internal Reflection (FTIR). In: 18th ACM Symposium on User Interface Software and Technology, pp. 115118. ACM Press, New York (2005)

4. Grossman, T., Wigdor, D.: Going Deeper:a Taxonomy of 3D on the Tabletop. In: IEEE International Workshop on Horizontal Interactive Human-Computer Systems (2007)

5. Hancock, M., Carpendale, S., Cockburn, A.: Shallow-depth 3D Interaction: Design and Evaluation of One-, Two- and Three-touch Techniques. In: ACM Conference on Human Factors in Computing Systems 2007, pp. 1147-1156. ACM Press, New York (2007)

6. Hancock, M., ten Cate, T., Carpendale, S.: Sticky Tools: Full 6 DOF Force-based Interaction for Multi-touch Tables. In: ACM Conference on Interactive TableTops and Surfaces, pp. 145-152. ACM Press, Banff (2009)

7. Kruger, R., Carpendale, S., Scott, S.D., Tang, A.: Fluid Integration of Rotation and Translation. In: ACM Conference on Human Factors in Computing Systems, pp. 601-610. ACM Press, New York (2005)

8. Reisman, J.L., Davidson, P.L., Han, J.Y.: A Screen-space Formulation for 2D and 3D Direct Manipulation. In: 22nd annual ACM symposium on User Interface Software and Technology, pp. 69-78. ACM Press, New York (2009)

9. Hilliges, O., Izadi, S., Wilson, A.D., Hodges, S., Mendoza, A.G., Butz, A.: Interactions in the Air: Adding Further Depth to Interactive Tabletops. In: 22nd ACM Symposium on User Interface Software and Technology, pp. 139-148. ACM Press, New York (2009)

10. Benko, H.: Beyond Flat Surface Computing: Challenges of Depth-aware and Curved Interfaces. In: 17th ACM International Conference on Multimedia, pp. 935-944 (2009)

11. Takeoka, Y., Miyaki, T., Rekimoto, J.: Ztouch: an Infrastructure for 3D Gesture Interaction in the Proximity of Tabletop Surfaces. In: ACM Conference on Interactive Tabletops and Surfaces, pp. 91-94. ACM Press, New York (2010)

12. Harrier, http: / / www.britannica.com/EBchecked/topic/255852/Harrier

13. Pierce, J.S., Forsberg, A.S., Conway, M.J., Hong, S., Zeleznik, R.C., Mine, M.R.: Image Plane Interaction Techniques in 3D Immersive Environments. In: ACM symposium on Interactive 3D Graphics, pp. 39-44. ACM Press, New York (1997) 